\section{Die arbeitgeberseitige Kündigung des Chefarztvertrages - Kündigungsgründe und Rechtsschutzmöglichkeiten (2. Teil)}

Der 1. Teil dieses Beitrages beschäftigte sich mit typischen Konstellationen, in denen es zur Kündigung eines Chefarztvertrages durch den Krankenhausträger kommen kann (vgl. Fortschr Röntgenstr 2018, DRGMitteilungen S. 199 ff.). Der hier folgende 2. Teil geht auf die Möglichkeiten ein, wie Chefärzte auf bevorstehende oder bereits ausgesprochene Kündigungen reagieren können. Er zeigt Rechtsschutzmöglichkeiten auf, stellt aber auch außergerichtliche Schlichtungsmöglichkeiten, wie z. B. eine Mediation vor.

\section{Außergerichtliche Streitbeilegung}

Die Kündigung eines Chefarztvertrages hat sowohl für den Chefarzt als auch für das Krankenhaus erhebliche Auswirkungen. Der Chefarzt verliert als Arbeitnehmer seinen Arbeitsplatz und aus einer gekündigten Anstellung ist es ihm - je nach Kündigungsgrund - erschwert, eine adäquate neue Anstellung zu finden. Die Kündigung eines Chefarztvertrages bedeutet aber auch für den Krankenhausträger als Arbeitgeber einen bedeutenden Einschnitt. Er verliert einen herausgehobenen Angestellten, mit dem er ggf. langfristig geplant hat, sowie dessen etwaige Zuweiserbeziehungen und auch dem Krankenhausträger kann es Probleme bereiten, adäquaten Ersatz für den üblicherweise hochqualifizierten und spezialisierten Chefarzt zu finden. Ein Kündigungsschutzprozess und seine Unwägbarkeiten sind für beide Vertragsparteien belastend. Für den Chefarzt gilt dies sowohl in persönlicher als auch in wirtschaftlicher Hinsicht. Er muss sich den Belastungen eines Gerichtsverfahrens aussetzen und wird während der laufenden Kündigungsschutzklage kein Gehalt von dem Krankenhausträger beziehen. Für den Krankenhausträger ergibt sich eine Belastung wohl vor allen Dingen in wirtschaftlicher Perspektive - er muss den „teuren“ Chefarzt ersetzen und gleichwohl damit rechnen, zur Weiterbeschäftigung des Chefarztes, zur Nachzahlung der bis zur gerichtlichen Entscheidung rückständigen Vergütung zzgl. Verzugszinsen sowie zur Zahlung eines Schadenersatzes für entgangene Liquidationseinnahmen oder zur Zahlung einer hohen Abfindung verpflichtet zu werden. Die Verhandlung vor dem Arbeitsgericht ist zudem öffentlich ( 52 S. 1 Arbeitsgerichtsgesetz (ArbGG)). Der Konflikt zwischen Krankenhausträger und Chefarzt würde deshalb ebenso öffentlich wie die gegenseitig erhobenen Vorwürfe. Eine solche Öffentlichkeit, die die Reputation des Chefarztes und des Krankenhausträgers gleichermaßen schädigen kann, werden beide Parteien üblicherweise vermeiden wollen.

\section{Kommunikation}

Um es erst gar nicht zu einer Situation kommen zu lassen, in der eine Kündigung ernsthaft im Raum stehen kann, empfiehlt es sich für beide Seiten, bei auftretenden Konflikten frühzeitig zu reagieren. Oftmals kann es bereits helfen, ein offenes und klärendes Gespräch zu führen. Die Erfahrung zeigt, dass auch eine professionelle und unabhängige Unterstützung von außen, z. B. durch einen Moderator oder einen Kommunikations-Coach, helfen kann, festgefahrene (Kommunikations-)Strukturen innerhalb eines Betriebes aufzulockern und den Parteien so zu einer für alle Beteiligten zufriedenstellenden Lösung zu verhelfen.

\section{Mediation}

Sofern die Probleme in einem Verhalten des Chefarztes liegen, das aus Sicht seines Arbeitgebers eine Kündigung des Arbeitsvertrages nicht als unausweichlich erscheinen lässt, kann zudem an ein Verfahren der außergerichtlichen Streitbelegung, insbesondere an eine Mediation nach dem Mediationsgesetz (MediationsG), gedacht werden. Die Vorschriften des MediationsG sind anwendbar, wenn die Konfliktparteien einen Mediator beauftragen, sie in einem strukturierten Verfahren bei der eigenverantworteten Lösung ihres Konflikts zu unterstützen. ${ }^{1}$

1 Greger, in: Greger/Unberath/Steffek, Recht der alternativen Konfliktlösung, 2. Aufl. 2016 , $\S 1 \mathrm{Rn} .6$.

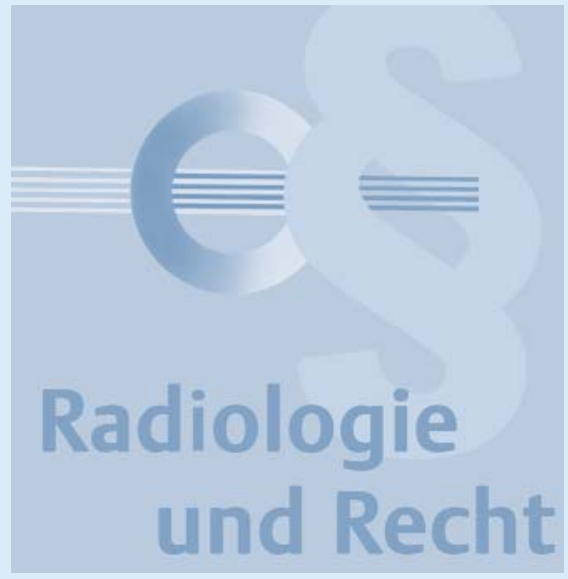

Ein Mediator ist eine unabhängige und neutrale Person ohne Entscheidungsbefugnis, die die Parteien durch die Mediation führt (§ 1 Abs. 2 MediationsG). Mediator im Sinne des MediationsG ist kein Beruf und kein geschützter Begriff. §5 Abs. 1 S. 1 MediationsG regelt, dass der Mediator in eigener Verantwortung durch eine geeignete Ausbildung und eine regelmäßige Fortbildung sicher stellt, dass er über theoretische Kenntnisse sowie praktische Erfahrungen verfügt, um die Parteien in sachkundiger Weise durch die Mediation führen zu können. Der Umfang der Ausbildung wird durch das MediationsG nicht geregelt. Es macht auch keine Vorgaben zu einer Ausbildungsstätte. $\S 5$ Abs. 1 S. 2 MediationsG benennt lediglich einige wesentliche Ausbildungselemente. ${ }^{2}$ Sofern die Tätigkeit des Mediators eine rechtliche Prüfung des Einzelfalls erfordert und er nicht nur durch rechtliche Regelungsvorschläge in die Gespräche der Beteiligten eingreift, sondern durch Ratschläge, Gestaltungsvorschläge, rechtliche Auskünfte und Bewertungen oder das Mitwirken an einer Abschlussvereinbarung Einfluss auf den Einigungsprozess der Beteiligten nimmt, ist die Vereinbarkeit seiner Tätigkeit mit dem Rechtsdienstleistungsgesetz im Blick zu behalten.

Ziel des Mediationsverfahrens, das üblicherweise vertraulich ist - §4 MediationsG verpflichtet die Beteiligten zur Verschwiegenheit -, ist die einvernehmliche Konfliktbeilegung. Durch den Mediator geführt, sollen die Beteiligten, die freiwillig an dem Mediationsverfahren teilnehmen, zu einer einvernehmlichen Konfliktlösung gelangen.

2 Greger, a. а. O., §5 Rn. $4 \mathrm{ff}$ 
Der Mediator hat dabei aber keine Entscheidungsbefugnis. Am Ende der Mediation soll nicht eine Entscheidung des Konflikts durch den Mediator, sondern vielmehr eine für alle Beteiligten zufriedenstellende Abschlussvereinbarung stehen.

Das aktive Hinwirken oder wenigstens die Bereitschaft des Chefarztes zur Teilnahme an einem Verfahren der außergerichtlichen Streitbeilegung kann auch im Hinblick auf die ultima-ratio-Funktion einer Kündigung empfehlenswert sein, weil dadurch seine Bereitschaft, die bestehenden Konflikte zu lösen und an der Situation zu arbeiten, zum Ausdruck kommt. Sollte es doch zu einer Kündigung des Arbeitsverhältnisses kommen, würde in die Abwägung, ob die Kündigung gerechtfertigt war oder ob ein Antrag des Krankenhausträgers auf Auflösung des Arbeitsverhältnisses nach $\S 9$ Abs. 1 S. 2 Kündigungsschutzgesetz (KSchG) begründet ist, mit Blick auf die Zumutbarkeit der Fortsetzung des Arbeitsverhältnisses für den Arbeitgeber, zugunsten des Chefarztes einzubeziehen sein, dass letzterer zur Veränderung der Situation und somit zur Schaffung der Voraussetzungen für eine Fortsetzung des Arbeitsverhältnisses bereit ist.

\section{Abmahnung}

Unabhängig von einer außergerichtlichen Streitbeilegung z. B. im Mediationsverfahren wird der Arbeitgeber den Chefarzt vor einer verhaltensbedingten Kündigung regelmäßig abmahnen müssen. Wegen der ultima-ratio-Funktion der Kündigung ist eine verhaltensbedingte Kündigung in der Regel unwirksam, wenn es an einer vorangegangenen einschlägigen Abmahnung fehlt. Die mit einer Abmahnung abgemahnte Pflichtverletzung eines Chefarztes kann jedoch nicht mehr zur Rechtfertigung einer Kündigung herangezogen werden. Mit der Abmahnung hat der Krankenhausträger zum Ausdruck gebracht, dass die vorgeworfene Pflichtverletzung eine Kündigung des Arbeitsverhältnisses nicht trägt. Insofern stellt sich für den Chefarzt die Frage, wie er auf eine Abmahnung reagiert. Einerseits hat er die Möglichkeit - sofern er die Abmahnung für unberechtigt hält -, ihre Entfernung aus seiner Personalakte zu verlangen und dies nötigenfalls einzuklagen.
Er kann aber auch eine Gegendarstellung zur Personalakte reichen. Der mit der Abmahnung gemachte Vorwurf kann eine Kündigung dann nicht mehr rechtfertigen. Mündet die Entwicklung schließlich doch in einem Kündigungsschutzprozess, kann der Chefarzt die Berechtigung der Abmahnung noch immer bestreiten.

\section{Freistellung}

Häufig kommt es im Vorlauf einer Kündigung, zugleich mit der Kündigung oder in deren Anschluss zu einer Freistellung des Chefarztes. Eine solche ist jedoch ohne eine besondere vertragliche Vereinbarung ${ }^{3}$ zwischen Krankenhausträger und Chefarzt oder ohne anerkennenswerten Grund ${ }^{4}$ nicht zulässig. Ein anerkennenswerter Grund liegt vor, wenn die Interessen des Krankenhausträgers an der tatsächlichen Nichtbeschäftigung des Chefarztes dessen Interessen an seiner Beschäftigung überwiegen. Das ist z. B. dann regelmäßig der Fall, wenn gegenüber dem Chefarzt Anhaltspunkte für das Begehen einer Straftat oder einer schweren Arbeitsvertragsverletzung (z. B. durch eine Konkurrenztätigkeit) bestehen, die der Krankenhausträger während der Freistellung aufklären möchte. Zugunsten des Chefarztes ist zu berücksichtigen, dass eine Freistellung eines Arbeitnehmers gegenüber Außenstehenden diskriminierenden Charakter haben kann. ${ }^{5}$ Insofern ist durch den Chefarzt zu bedenken, ob er sich gegen eine Freistellung vor Ablauf der Kündigungsfrist zur Wehr setzen sollte. Kommt er zu dem Entschluss dies zu tun, muss er den Erlass einer einstweiligen Verfügung beim Arbeitsgericht erwirken.

\section{Kündigungsschutzklage}

Entschließt sich der Krankenhausträger schließlich zur Kündigung des Arbeitsverhältnisses mit seinem Chefarzt, muss letzterer, wenn er sich gegen die Kündigung zur Wehr setzen möchte, zügig und überlegt handeln.

3 BAG, Urt. v. 21.9.1993, Az.: 9 AZR 335/91.

4 LAG Köln, Beschl. v. 20.3.2001, Az.: 6 Ta 46/01.

5 Koch, in: Schaub, Arbeitsrechtshandbuch, 13. Aufl. 2009, §110 Rn. 10.

\section{Klagefrist}

Gleich, ob ein Arbeitgeber ein Arbeitsverhältnis ordentlich oder außerordentlich nach §626 Abs. 1 Bürgerliches Gesetzbuch (BGB) kündigt, sieht §4 S. 1 KSchG vor, dass ein Arbeitnehmer, der geltend machen will, dass eine Kündigung sozial ungerechtfertigt oder aus anderen Gründen rechtsunwirksam ist, innerhalb von drei Wochen nach Zugang der Kündigung Klage beim Arbeitsgericht auf Feststellung erheben muss, dass das Arbeitsverhältnis durch die Kündigung nicht aufgehoben ist.

\section{Formale Mängel}

In jedem Fall sollte der Chefarzt zunächst prüfen lassen, ob die formalen Voraussetzungen einer wirksamen Kündigung vorliegen. Ist dies nicht der Fall, würde das Arbeitsgericht auch ohne Prüfung der materiellen Kündigungsvoraussetzungen, also der sozialen Rechtfertigung (vgl. § 1 KSchG) oder eines wichtigen Grundes (vgl. $\S 626$ Abs. 1 BGB), den Fortbestand des Arbeitsverhältnisses feststellen.

Nach §623 BGB ist eine Kündigungserklärung nur dann wirksam, wenn sie schriftlich erfolgt. Eine Kündigung durch Fax oder E-Mail ist damit ausgeschlossen. Die Kündigung wird wirksam mit ihrem Zugang bei dem Empfänger und ist grundsätzlich bedingungsfeindlich. Sie muss inhaltlich bestimmt sein und die Auflösung des Arbeitsverhältnisses zumindest sinngemäß zum Ausdruck bringen.

Unabhängig davon, ob der Krankenhausträger privat, öffentlich oder freigemeinnützig ist, ist vor jeder Kündigung regelmäßig die Mitarbeitervertretung anzuhören, jedenfalls sofern der Chefarzt kein leitender Angestellter im Sinne der jeweiligen Regelungen ist. Das ergibt sich aus $\S 102 \mathrm{Be}-$ triebsverfassungsgesetz bzw. $§ 79$ Abs. 1 Bundespersonalvertretungsgesetz für private bzw. öffentliche Krankenhausträger sowie aus $\S \S 42,46$ Mitarbeitervertretungsgesetz der EKD bzw. aus §§ 30, 31 Mitarbeitervertretungsordnung für evangelische bzw. katholische Krankenhausträger. Dabei sind durch den Krankenhausträger alle Umstände mitzuteilen, deren Kenntnis erforderlich ist, um eine Stellungnahme zu der beabsichtigten Kündigung abgeben zu können. 
Eine ordentliche Kündigung kann auch (tarif-)vertraglich ausgeschlossen sein, sodass nur eine außerordentliche Kündigung in Betracht kommt. Für die außerordentliche Kündigung gilt nach $\S 626$ Abs. 2 BGB eine zweiwöchige Kündigungserklärungsfrist ab dem Zeitpunkt, in dem der Kündigungsberechtigte von den für die Kündigung maßgebenden Tatsachen Kenntnis erlangt.

\section{Verfahren vor dem Arbeitsgericht}

Die mündliche Verhandlung vor dem $\mathrm{Ar}$ beitsgericht beginnt mit einer Güteverhandlung (§54 Abs. 1 S. 1 ArbGG). Diese findet regelmäßig innerhalb weniger Wochen nach Eingang der Kündigungsschutzklage bei dem Arbeitsgericht statt. In dem Gütetermin wird das Gericht versuchen, noch einmal eine Einigung zwischen Krankenhausträger und Chefarzt herbeizuführen.

Seit der Einführung des $\S 54$ Abs. 6 ArbGG am 26.07.2012 ${ }^{6}$ kann der vorsitzende Richter die Parteien für die Güteverhandlung sowie deren Fortsetzung vor einen hierfür bestimmten und nicht entscheidungsbefugten Richter desselben Gerichts (Güterichter) verweisen. Der Güterichter kann alle Methoden der Konfliktbeilegung einschließlich der Mediation einsetzen. Die Verweisung kann nur bis zum Scheitern der Güteverhandlung und nur mit dem Einverständnis der Parteien erfolgen. Die Verhandlung vor dem Güterichter ist - anders als die normale mündliche Verhandlung - nicht öffentlich und unterliegt keinen besonders normierten Verfahrensvorschriften. Ein vor dem Güterichter abgeschlossener Vergleich beendet wie ein gerichtlicher Vergleich das Verfahren und ist zugleich Vollstreckungstitel. ${ }^{7}$ Nach §54a Abs. 1 ArbGG kann das Gericht den Parteien zudem eine Mediation oder ein anderes Verfahren der außergerichtlichen Streitbeilegung vorschlagen. Entscheiden sich die Parteien dazu, ordnet das Gericht zunächst das Ruhen des Verfahrens an (§54a Abs. 2 ArbGG).

Kommt eine einvernehmliche Beendigung des Kündigungsschutzprozesses zwischen

$6 \quad$ Art. 4 des MediationsGEG v. 21.07.2012, BGBI.I S. 1577.

7 Koch, in: Erfurter Kommentar zum Arbeitsrecht, 17. Aufl. 2017, §54 ArbGG, Rn. 11. den Parteien in der Güteverhandlung und in dem Verfahren vor dem Güterichter nicht zustande, wird das Gericht einen Kammertermin anberaumen, der üblicherweise einige Wochen oder sogar Monate nach dem Gütetermin stattfindet. Das Gericht wird dann das Vorliegen sämtlicher Voraussetzungen der Kündigung prüfen. Sofern die Klage nicht bereits aufgrund formaler Fehler der Kündigung erfolgreich sein muss, wird es sich bei einer verhaltensbedingten Kündigung insbesondere mit dem vermeintlichen Fehlverhalten des Chefarztes und einer Abwägung der Interessen von Krankenhausträger und Chefarzt befassen.

Entscheidet das Arbeitsgericht zugunsten des Chefarztes und stellt fest, dass das Arbeitsverhältnis durch die Kündigung des Krankenhausträgers nicht aufgelöst worden ist, hat der Chefarzt einen Anspruch auf Nachzahlung seiner rückständigen Vergütung, die nach §288 Abs. 1 BGB mit fünf Prozentpunkten über dem jeweiligen Basiszinssatz zu verzinsen ist. Ist dem Chefarzt ein eigenes Liquidationsrecht eingeräumt worden, kann er zudem Schadenersatz für die entgangenen Liquidationseinnahmen, die er bei durchgehender Beschäftigung gehabt hätte, verlangen. ${ }^{8}$

Allerdings kann der Krankenhausträger, auch wenn die Kündigungsschutzklage des Chefarztes wegen einer fehlenden sozialen Rechtfertigung der Kündigung erfolgreich ist und das Arbeitsgericht feststellt, dass das Arbeitsverhältnis durch die Kündigung des Krankenhausträgers nicht aufgelöst worden ist, u. U. dennoch die Beendigung des Arbeitsverhältnisses erreichen. Er kann nach § 9 Abs. 1 S. 2, 3 KSchG bis zum Schluss der letzten mündlichen Verhandlung in einer etwaigen Berufungsinstanz den Antrag stellen, das Arbeitsverhältnis gegen Zahlung einer Abfindung aufzulösen. Voraussetzung für den Erfolg des Antrags ist, dass Gründe vorliegen, die eine den Betriebszwecken dienliche weitere Zusammenarbeit zwischen Arbeitgeber und Arbeitnehmer nicht erwarten lassen. Die Anforderungen an die Antragsbegründung sind streng. ${ }^{9}$ Weil es hinsichtlich dieser Gründe aber auf ein Verschulden des

8 BAG, Urt. v. 15.9.2011, Az.: 8 AZR 846/09.

9 BAG, Urt. v. 29.8.2013, Az.: 2 AZR 419/12.
Chefarztes nicht ankommt, da die Abfindung durch $\S 10$ KSchG der Höhe nach begrenzt ist und weil die Arbeitsgerichte eher geringe Abfindungen zusprechen, birgt die Möglichkeit eines solchen Auflösungsantrages durch den Krankenhausträger ein nicht zu vernachlässigendes Risiko für den Chefarzt.

Der Antrag kann bspw. auch mit dem Verhalten des Chefarztes nach dem Beginn der Konflikte mit dem Krankenhausträger begründet werden. So soll auch die fehlende Einsichtsfähigkeit eines Arbeitnehmers einen Fehler begangen zu haben, einer den Betriebszwecken dienlichen weiteren Zusammenarbeit entgegenstehen, wenn die fehlende Einsichtsfähigkeit auf eine Gefahr der Wiederholung der Pflichtverletzung schließen lässt. ${ }^{10}$ Auch insofern kann der Bereitschaft des Chefarztes zur außergerichtlichen Streitbeilegung noch in dieser späteren Phase der Auseinandersetzung Bedeutung zukommen. Ein besonderes Risiko trägt der Chefarzt, der ausnahmsweise leitender Angestellter im Sinne des $\S 14$ Abs. 2 KSchG ist. Ist dies der Fall, bedarf der Antrag des Krankenhausträgers auf Auflösung des Arbeitsverhältnisses keiner Begründung (§ 14 Abs. 2 S. 2 KSchG). Unstatthaft ist der Antrag hingegen, wenn die Kündigungsschutzklage bereits aus formalen Gründen erfolgreich ist oder wenn nur eine Kündigung des Arbeitsverhältnisses aus wichtigem Grund nach $\S 626$ Abs. 1 BGB in Betracht kommt.

\section{Vergleich}

Die Parteien haben jederzeit die Möglichkeit, die Auseinandersetzung durch einen Vergleich zu beenden. Dieser wird regelmäßig einen Aufhebungsvertrag und eine Abfindungszahlung an den Chefarzt vorsehen. Die Höhe der Abfindung ist in einem solchen Fall zwischen dem Krankenhausträger und dem Chefarzt grundsätzlich frei verhandelbar und hängt vom Einzelfall ab. Dabei steigt mit der Dauer eines gerichtlichen Verfahrens der wirtschaftliche Druck auf den Krankenhausträger. Er trägt das Risiko, dem Chefarzt bei einem Unterliegen eine rückständige Vergütung und Schadenersatz für entgangene Liquidationseinnah-

10 LAG Köln, Urt. v. 19.4.2005, Az.: 9 (6) Sa 1059/04. 
men zahlen sowie den Chefarzt während der Dauer der Kündigungsstreitigkeit ersetzen zu müssen. Der Chefarzt hätte bei einer Abweisung der Kündigungsschutzklage keinen Anspruch auf eine Weiterbeschäftigung und keinen Anspruch auf die Zahlung einer Abfindung. Sollte der Krankenhausträger erfolgreich den Antrag auf Auflösung des Arbeitsverhältnisses nach §9 Abs. 1 S. 2 KSchG stellen, erhielte der Chefarzt lediglich eine Abfindung nach $\S 10$ KSchG. Insofern ist dem Chefarzt zu raten, zusammen mit seinem Rechtsberater zu jeder Zeit seine Erfolgsaussichten und Chancen sowie Risiken zu prüfen, um zu dem richtigen Zeitpunkt einen für ihn möglichst günstigen Vergleich mit dem Krankenhausträger schließen zu können.

\section{Zusammenfassung}

Die Beendigung des Chefarztvertrages durch eine Kündigung des Krankenhausträ- gers ist für alle Beteiligten mit Belastungen und Risiken verbunden. Es liegt deshalb regelmäßig in dem Interesse beider Parteien, es gar nicht zu einer solchen Situation kommen zu lassen. Oftmals können bestehende Konflikte bereits durch eine Veränderung der Kommunikation oder mit Hilfe eines Mediators gelöst werden. Gelingt dies nicht, muss der Chefarzt stets prüfen, wie er auf die verschiedenen Schritte des Krankenhausträgers (Abmahnung, Freistellung, Kündigung) reagiert und wie er sich auch gerichtlich erfolgreich dagegen wehren kann. Insbesondere wenn eine Kündigung ausgesprochen wurde, ist ein zügiges und wohlüberlegtes Handeln des Chefarztes geboten. Aber auch zuvor können unüberlegte und falsche Entscheidungen oder Äußerungen des Chefarztes seine Position in der Auseinandersetzung erheblich verschlechtern. Ob und unter welchen Bedingungen er ggf. bereit ist, sich mit dem Krankenhausträger zu vergleichen, sollte er zu jeder Zeit bedenken und anhand seiner Erfolgsaussichten in dem Klageverfahren sowie der individuellen Chancen und Risiken abschätzen lassen, um zum richtigen Zeitpunkt in der Auseinandersetzung den für ihn besten Vergleich erwirken zu können.

Prof. Dr. Peter Wigge

Rechtsanwalt

Fachanwalt für Medizinrecht

Jonas Kaufhold

Rechtsanwalt

Rechtsanwälte Wigge

Scharnhorststraße 40

48151 Münster

Telefon: (0251) $53595-0$

Telefax: (0251) 53595 - 99

E-Mail: kanzlei@ra-wigge.de www.ra-wigge.de 